\title{
Structural Studies of Ebola Viral Homolog Encoded by Microbats
}

\author{
Garrett M. Ginell ${ }^{1}$, Gaya K. Amarasinghe ${ }^{2}$, Christopher F. Basler ${ }^{3}$, Lisa J. Keefe ${ }^{4}$ and Daisy W. \\ Leung $^{2}$ \\ ${ }^{1}$ Biochemistry and Molecular Biology Department, Cornell College, Mt. Vernon, IA 52314 \\ ${ }^{2}$ Department of Pathology and Immunology, Washington University School of Medicine, St. Louis, MO \\ 63110, USA \\ ${ }^{3}$ Center for Microbial Pathogenesis, Institute for Biomedical Sciences, Georgia State University, Atlanta, \\ GA 30303 \\ ${ }^{4}$ Industrial Macromolecular Crystallography Association - Collaborative Access Team, Hauptman- \\ Woodward Medical Research Institute, Buffalo, New York 14203
}

High case fatality rates are observed during sporadic Ebola virus outbreaks. Yet Ebola the reservoir host or safe an efficacious treatments are currently not available. Bats have been identified as one potential reservoir host, but why Ebola is highly virulent in some mammals and not in others is not well understood. In an effort to address this gap in our knowledge, we have initiated a study to test the role of filoviral genes that are preserved in some mammalian genomes, including those of bats. Filoviral genomes encode seven structural proteins, one of which is viral protein 35 (VP35), a multifunctional protein that both enhances viral propagation in Ebola and inhibits the immune response in its host. VP35 antagonizes type I interferon production by targeting RNA-dependent and RNA-independent mechanisms. Bats have unique features that may potentially support a symbiotic relationship with viruses that enables them to act as a reservoir host for zoonotic viruses such as Ebola. VP35 protein in bats (batVP35), which has been conserved over millions of years, is thought to be a key component aiding in protection from Ebola infection. As a part of the efforts to better understand if VP35 confers resistance to the virus in bats, structural studies of the bat VP35 have been undertaken. Significant challenges were encountered in solving the crystal structure. A mutated limited sequence of bat VP35 was used to crystallize the protein. While extensive screening of conditions produced a few single crystals, only one of the crystals was suitable for diffraction experiments. Thus, only a single native data set was collected. Data were measured at the IMCA-CAT insertion device beamline 17-ID at the Advanced Photon Source. The threedimensional atomic structure of bat viral protein VP35 was solved by molecular replacement using a model of only $33 \%$ homology. The structure has been refined to $2.50 \AA$ resolution. Comparison of the structure of this bat VP35 protein with that of the Ebola VP35 protein reveals structural similarity to the viral VP35. However, batVP35 also shows unusual biophysical properties as well as dsRNA binding and IFN inhibition, which collectively show significant functional differences that may contribute to its role in bat cells. We will discuss major differences and potential implications to viral replication and host responses as well as potential new targets for therapeutic development. 